\title{
Melt front propagation in dielectrics upon femtosecond laser irradiation: Formation dynamics of a heat-affected layer
}

Mario Garcia-Lechuga, Javier Solis, and Jan Siegel

Citation: Appl. Phys. Lett. 108, 171901 (2016); doi: 10.1063/1.4948262

View online: http://dx.doi.org/10.1063/1.4948262

View Table of Contents: http://aip.scitation.org/toc/apl/108/17

Published by the American Institute of Physics

\section{Articles you may be interested in}

Exceptionally crystalline and conducting acid doped polyaniline films by level surface assisted solution casting approach

Appl. Phys. Lett. 108, 161901161901 (2016); 10.1063/1.4947083

Ru nucleation and thin film smoothness improvement with ammonia during chemical vapor deposition Appl. Phys. Lett. 34, 031508031508 (2016); 10.1116/1.4944852

Nanoscale current spreading analysis in solution-processed graphene oxide/silver nanowire transparent electrodes via conductive atomic force microscopy

Appl. Phys. Lett. 119, 195501195501 (2016); 10.1063/1.4949502
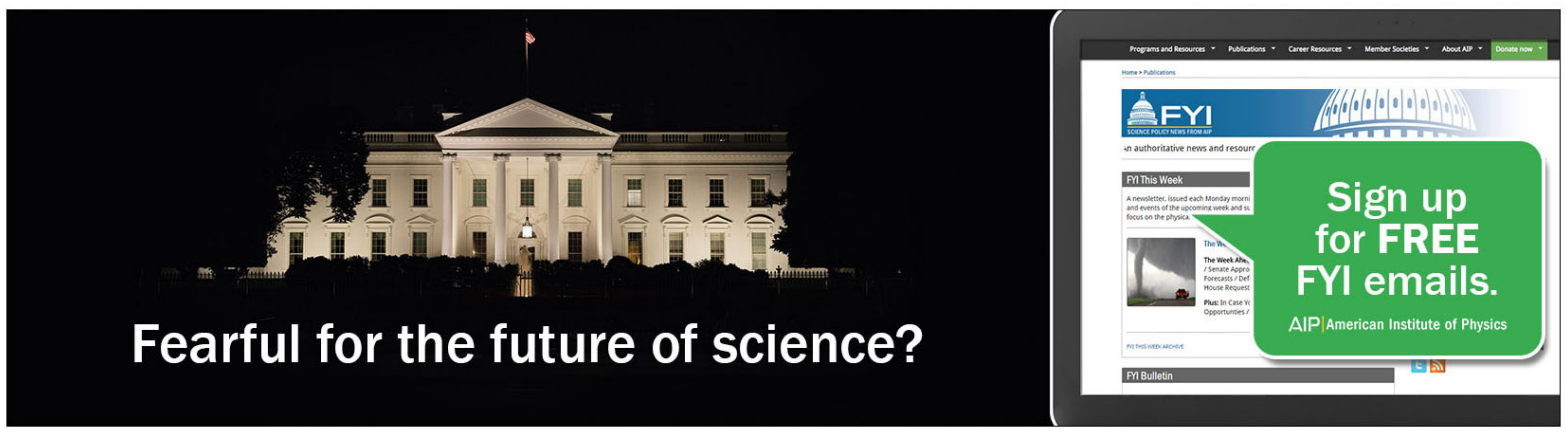


\title{
Melt front propagation in dielectrics upon femtosecond laser irradiation: Formation dynamics of a heat-affected layer
}

\author{
Mario Garcia-Lechuga, ${ }^{\text {a) }}$ Javier Solis, and Jan Siegel ${ }^{\mathrm{a})}$ \\ Laser Processing Group, Instituto de Optica, CSIC, Serrano 121, 28006 Madrid, Spain
}

(Received 15 March 2016; accepted 12 April 2016; published online 26 April 2016)

\begin{abstract}
Several studies in dielectrics have reported the presence of a thin heat-affected layer underneath the ablation crater produced by femtosecond laser irradiation. In this work, we present a timeresolved microscopy technique that is capable of monitoring the formation dynamics of this layer and apply it to the study of a phosphate glass exposed to single pulses below the ablation threshold. A few nanoseconds after laser excitation, a melt front interface can be detected, which propagates into the bulk, gradually slowing down its speed. By means of image analysis combined with optical modeling, we are able to determine the temporal evolution of the layer thickness and its refractive index. Initially, a strong transient decrease in the refractive index is observed, which partially recovers afterwards. The layer resolidifies after approximately $1 \mu \mathrm{s}$ after excitation, featuring a maximum thickness of several hundreds of nanometers. Published by AIP Publishing.

[http://dx.doi.org/10.1063/1.4948262]
\end{abstract}

Amplified femtosecond (fs) laser systems have proven to be a powerful tool for micro- and nano-structuring of materials. Especially important is their use in processing of transparent materials, ${ }^{1}$ since the high peak intensities involved allow triggering of non-linear absorption, generating free electrons. The density of free carriers can be further increased by secondary processes, such as impact ionization. ${ }^{2,3}$ If the critical density is exceeded, optical breakdown occurs and material ejection is produced, ${ }^{4}$ generally leading to smoother craters than those produced with longer pulses.

Because of the ultrashort duration of fs-pulses, the optical energy is entirely deposited in the electron system, and the structural changes occur only after electrons have transferred their energy to the lattice. This is the reason why the volume surrounding the crater, commonly known as heataffected zone (HAZ) or heat-affected layer (HAL), is considerably reduced compared to the case of longer pulses. ${ }^{5}$ However, thermal effects caused by the ultrashort pulses should not be overlooked, especially in strong excitation conditions, where the amount of energy transferred by the expanding ablating plasma to the underneath layer is very large, affecting regions that have not been optically excited. The importance of thermal effects after irradiation with single fs-laser pulses was highlighted by Ben-Yakar et al., ${ }^{6}$ who demonstrated the existence of a thin, transiently melted layer below the surface, showing it to be responsible for the formation of a rim surrounding the ablation crater in a borosilicate glass. Also, by means of a model based on the heat diffusion equation, the authors simulate the propagation dynamics of the melt interface into the bulk. In a recent work by our group, we have introduced an experimental method, based on optical microscopy $(\mathrm{OM})$ with monochromatic light illumination, capable of quantifying the final refractive index change and thickness of the heat-affected layer (HAL) underneath the ablation crater. ${ }^{7}$ Indeed, the direct proof of

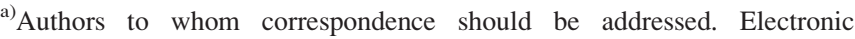
addresses: mario@io.cfmac.csic.es and j.siegel@io.cfmac.csic.es
}

the formation of a HAL has been provided in a variety of dielectric materials, including organic (polymers) and inorganic (crystalline and glassy) materials. ${ }^{7-9}$

However, there is a lack of experimental studies aimed at investigating the formation process of the HAL. In the present work, we have added temporal resolution to the optical microscopy system with monochromatic illumination used in our earlier work to quantify the layer thickness and properties ${ }^{7}$ and adapted it to a laser irradiation configuration to allow in-situ monitoring of processes happening up to hundreds of nanoseconds after irradiation. We have employed pump-probe microscopy, ${ }^{10}$ in which a delayed probe pulse is used for illumination in a microscope configuration, providing ultrashort temporal and micrometric spatial resolution. This technique allows the direct observation of a hot viscous layer expanding into phosphate glass, enabling the characterization of its transient properties and solidification time.

Figure 1 shows the experimental set-up. The laser system is a Ti:sapphire femtosecond amplifier that produces pulses of 120 fs FWHM (Full Width at Half Maximum) centered at $800 \mathrm{~nm}$ wavelength at a repetition rate of $100 \mathrm{~Hz}$. A mechanical shutter allows selecting a single pulse, which is split into two: pump and probe. The pump pulse is s-polarized and focused at the sample surface at an angle of $53^{\circ}$ exciting an elliptical region with a measured Gaussian intensity distribution $\left(59 \mu \mathrm{m} \times 97 \mu \mathrm{m} \mathrm{1} / \mathrm{e}^{2}\right.$-diameters). ${ }^{11}$ The pulse energy is controlled by a combination of half wave plate and polarizing cube beamsplitter and monitored with a reference photodiode (PD).

The probe pulse is frequency-doubled to $400 \mathrm{~nm}$ and used to illuminate the surface sample, which is imaged by means of an objective $(80 \times$, N.A $=0.45)$ and tube lens onto a charge-coupled device (CCD) camera. We use $400 \mathrm{~nm}$ probe light instead of $800 \mathrm{~nm}$ in order to increase the spatial resolution of the microscope. In order to study the thermal effects up to the $\mu$ s time scale, ${ }^{6}$ an optical delay configuration in $\mathrm{air}^{3}$ is unpractical as it would require hundreds of 


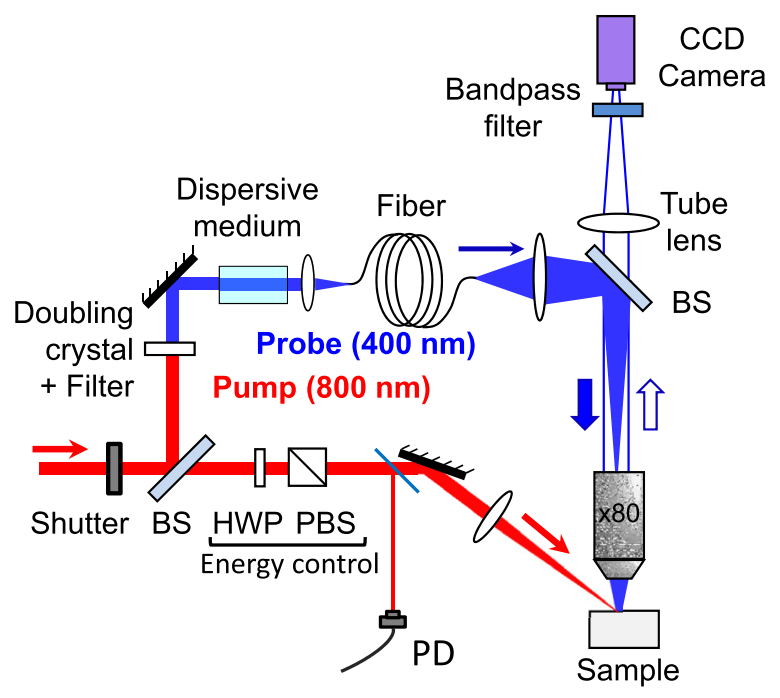

FIG. 1. Sketch of the irradiation and time-resolved microcopy setup for long delays. BS: beam splitter; PBS: polarizing beam splitter, HWP: half-wave plate; PD: photodiode; and CCD: charge-coupled device (Camera).

meters of optical path. To this end, we have developed a plug-and-play optical fiber delay line based on multimode fiber patchcords with moderate energy attenuation over a broad optical bandwidth (250-1200 nm). In order to avoid white light generation/damage in the fiber, the laser pulses were stretched before injection using a $15 \mathrm{~cm}$ long borosilicate glass block. The fiber patchcords with different lengths can be connected, enabling to flexibly cover a delay window up to $0.5 \mu \mathrm{s}$. Compared to other approaches for covering long-range delays (like using two different laser sources), our approach is almost jitterfree and avoids synchronization problems.

The sample studied in this work is an undoped phosphate glass (MM2 by Kigre), chosen mainly because of two interesting properties. First, a relatively thick permanent HAL is formed upon irradiation with a single fs-laser pulse. ${ }^{7}$ Second, it features surface swelling at fluences just below the ablation threshold $\left(\mathrm{F}_{\mathrm{th}}=3.8 \mathrm{~J} / \mathrm{cm}^{2}\right)$, offering an ideal fluence window for studying the formation and evolution of the HAL without being shielded by ablation effects. ${ }^{7,12}$

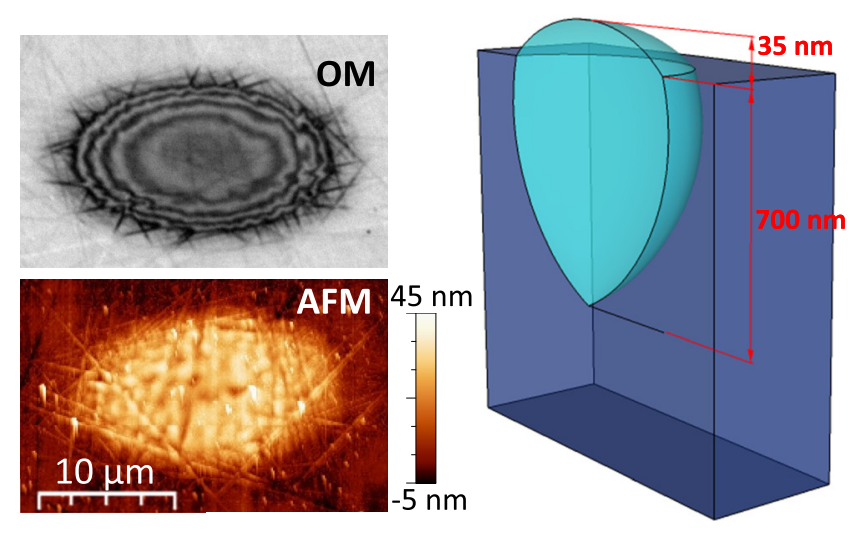

FIG. 2. (Right) 3D-representation (not to scale) of a transversal section of the heat-affected structure formed in phosphate glass after single pulse fslaser irradiation of the sample surface. (Left) Optical microscope (460 nm wavelength illumination, $100 \times$ objective lens, and N.A. $=0.9)$ and atomic force microscope images of the sample surface after irradiation.
Figure 2 shows the effect induced by a single pulse at a fluence of $3.7 \mathrm{~J} / \mathrm{cm}^{2}$, slightly below the ablation threshold. The optical microscopy (OM) image shows the appearance of Newton rings. This effect occurs when monochromatic light reflected by the upper and lower interfaces of the HAL interferes. For a complete characterization of the HAL shape, a measurement of the surface topography by Atomic Force Microscopy (AFM, Agilent 5100) was taken, featuring in this case swelling of the surface up to $35 \mathrm{~nm}$, without signs of ablation. Based on these two measurements, the refractive index and thickness distribution of the HAL can be obtained by analyzing the number, spacing, and optical contrast of the rings, using optical modeling based on Fresnel equations. The result is shown as a 3D-representation in Figure 2. Two remarks about the scale of the modification produced should be made. First, the depth extension is around 20 times larger that the surface swelling. Second, the lateral dimensions of the HAL are considerably larger $(\sim 20 \mu \mathrm{m})$ than its maximum thickness $(\sim 700 \mathrm{~nm})$.

Figure 3 displays several time-resolved images of the surface at different delays using the system shown in Figure 1. Each image was recorded on a fresh region of the sample exposed to a single pulse. The three shorter time delays were obtained by using an optical delay line in air, ${ }^{3}$ the others by using the fiber delay-line. At $t=750 \mathrm{fs}$, a marked increase in surface reflectivity (by a factor $\sim 2$ ) can be observed, which is a characteristic sign of free carrier generation via non-linear absorption. Using a simple Drude model, a maximum carrier density of $n_{e}=1.1 \times 10^{22} \mathrm{~cm}^{-3}$ can be estimated. ${ }^{12}$ At $t=20$ ps, the reflectivity has decreased below that of the non-excited material (by a factor $\sim 0.5$ ). While this behavior might be interpreted as a sign of ablation, it should be kept in mind that the fluence employed is below the ablation threshold (see the AFM image in Fig. 2). This implies that the transient reflectivity decrease must have a different origin, most likely the formation of a thin layer of hot fluid at solid density after isochoric heating. ${ }^{13}$ The transient decrease in reflectivity gradually recovers within hundreds of picoseconds. At $t=1.5 \mathrm{ns,}$ the first sign of a Newton ring structure appears; yet, the image quality does not allow a precise analysis and quantitative analysis.

In contrast, at $t=11.2 \mathrm{~ns}$, it is possible to observe and count the number of Newton rings: 4 minima and 3 maxima. The image quality is still compromised by a grainy texture caused by speckle, which is an inherent problem when using a spatially coherent light source for illumination (even for ultrafast lasers with relatively short coherence lengths). Speckle is gradually reduced as the fiber length is increased, leading to better quality images. A careful inspection of the images at increasing delays ( $t=78 \mathrm{~ns}, 128 \mathrm{~ns}$, and $434 \mathrm{~ns})$ reveals that the number of Newton rings increases. The last image, recorded $1 \mathrm{~s}$ after irradiation, features 6 minima and 5 maxima. Table I displays the evolution of the optical thickness $d$ with delay time, directly extracted by counting the number of rings (\#, irrespective of being maximum or minimum) and considering illumination wavelength $\lambda$ as $d=\# \cdot \lambda$ / 4. It is worth emphasizing at this point that the time window in which this kind of Newton rings is observed is $100 \times$ larger than the narrow window in which similar rings have 

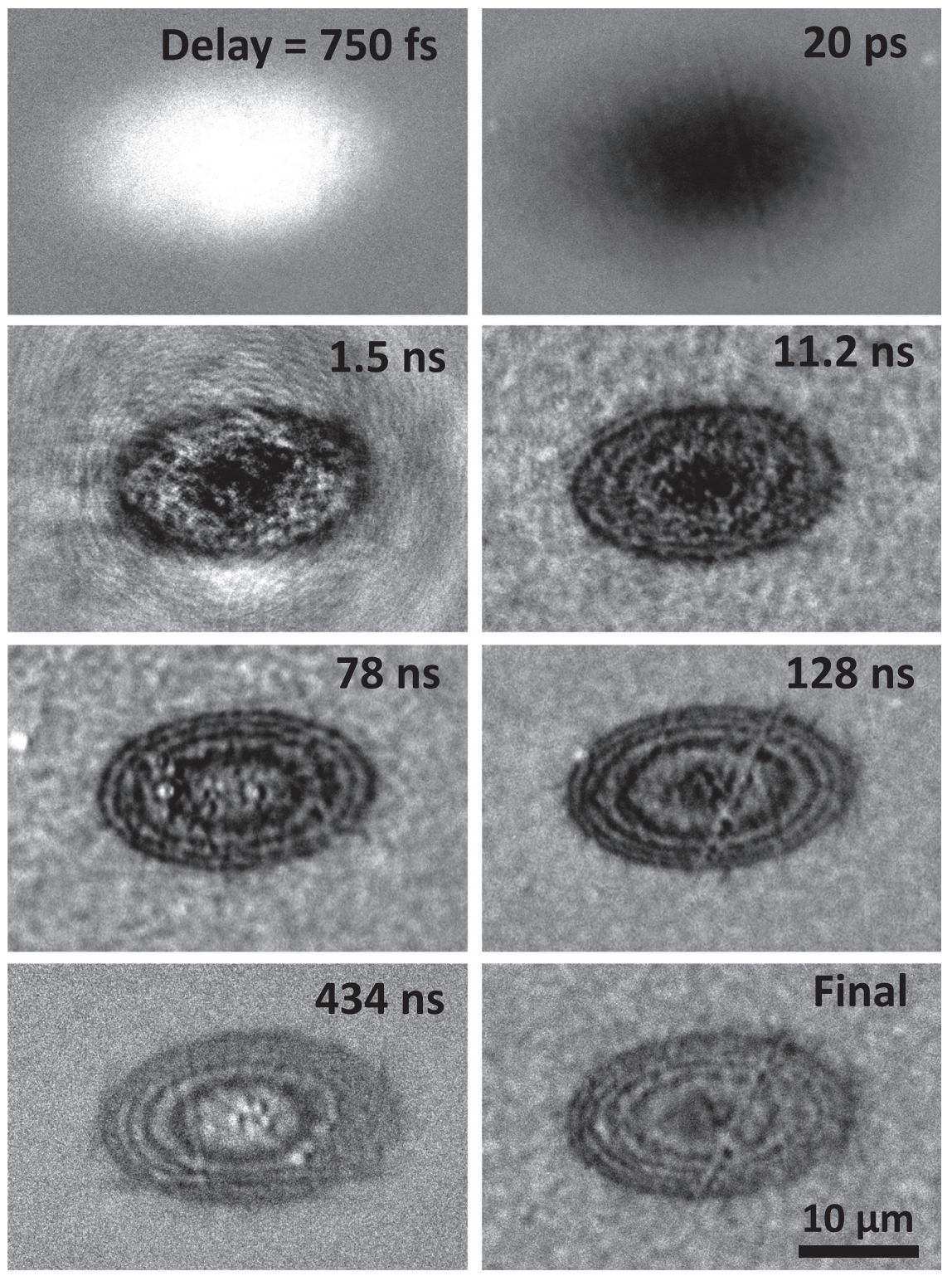

FIG. 3. Time-resolved microscopy images ( $400 \mathrm{~nm}$ illumination) of the sample surface at different time delays after irradiation (time labelled). The grey scale bounds are set to $[0.5,1.5]$ for the first two images and to $[0.8$, $1.2]$ for the others. been observed in some dielectrics above the ablation threshold, caused by the expansion of an ablating layer. ${ }^{13}$

An analysis of the images in Figure 2 reveals that the interference minima for a given delay have approximately the same reflectivity value (e.g., $\mathrm{R}_{\min }=0.82$ at $t=128 \mathrm{~ns}$ ) and so do the maxima (for all delays $\mathrm{R}_{\max }=1$, except the central disc at $t=434 \mathrm{~ns}$ ). Using these experimental values, we have

TABLE I. Data extracted from time-resolved images (2nd and 3rd columns) and data obtained by optical modelling (4th and 5th columns).

\begin{tabular}{lcccc}
\hline \hline Time after & Max. optical & $\begin{array}{c}\text { Relative } \\
\text { reflectivity } \\
\text { irradiation (ns) }\end{array}$ & $\begin{array}{c}\Delta \mathrm{n} / \mathrm{n}_{0} \\
\left(\times 10^{-2}\right)\end{array}$ & $\begin{array}{c}\text { HAL } \\
\text { thickness (nm) }\end{array}$ \\
\hline $\mathrm{R}_{\max } / \mathrm{R}_{\min }$ & $(\mathrm{nm})$ \\
\hline 11.2 & 700 & $1 / 0.78$ & $-2.5 \pm 0.2$ & $460 \pm 20$ \\
26.5 & 750 & $1 / 0.80$ & $-2.3 \pm 0.2$ & $490 \pm 20$ \\
78 & 800 & $1 / 0.80$ & $-2.3 \pm 0.2$ & $520 \pm 20$ \\
128 & 850 & $1 / 0.82$ & $-2.1 \pm 0.3$ & $590 \pm 20$ \\
256 & 920 & $1 / 0.82$ & $-2.1 \pm 0.3$ & $625 \pm 20$ \\
358 & 950 & $1 / 0.85$ & $-1.8 \pm 0.3$ & $640 \pm 20$ \\
434 & 1000 & $1 / 0.85$ & $-1.8 \pm 0.3$ & $670 \pm 20$ \\
Final & 1100 & $1 / 0.87$ & $-1.4 \pm 0.4$ & $720 \pm 20$ \\
\hline \hline
\end{tabular}

used a multilayer model based on Abeles theory ${ }^{14}$ to determine, for each delay, the thickness and refractive index distribution. As for the latter, we could discard the possibility of a gradient-index layer based on the experimental data. The best match to the data was obtained by assuming a homogeneous layer with a reduced refractive index $n$ and an unchanged absorption coefficient $(k=0)$. The transient thickness and refractive index values of the HAL obtained from these calculations are shown in Table I. The original glass refractive index is $n_{0}(400 \mathrm{~nm})=1.556$.

Since the refractive index changes are negative in all cases, it is most likely that the density of the HAL is reduced compared to the original glass, which is consistent with the presence of surface swelling after irradiation. Assuming a linear relationship between the refractive index $n$, material density $\rho$, and thickness $d$ of the HAL as proposed in Ref. 15, we would expect a surface swelling of $\Delta d=+10 \mathrm{~nm}$, according to the relationship $\Delta n / n_{0}=-0.014=\Delta \rho / \rho_{0}=-\Delta d / d$. The considerably higher value measured experimentally $(\Delta d=+35 \mathrm{~nm})$ suggests that this phenomenological relation has a limited validity. Considerably lower $\Delta n / n$ values are observed for short delays (e.g., $\Delta n / n_{0}=-0.025$ at $11.2 \mathrm{~ns}$ ), leading to a better 


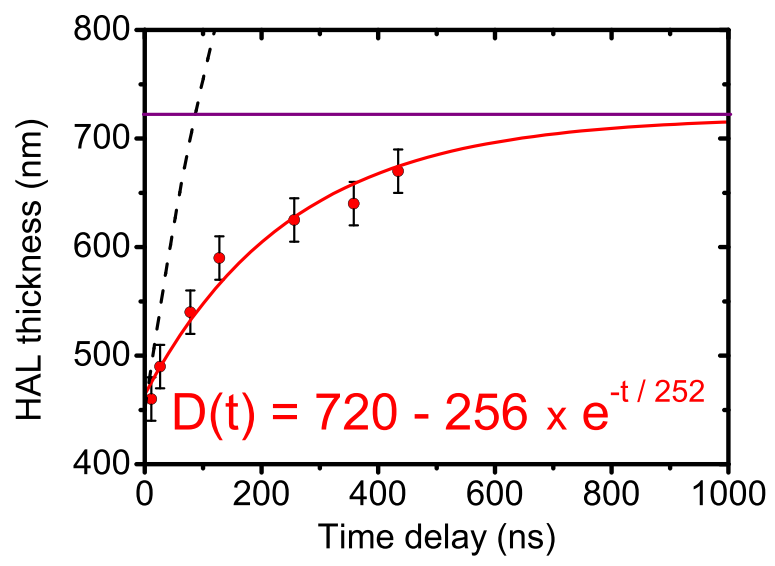

FIG. 4. Experimental (symbols) of maximum thickness of the heat-affected layer as a function of time, fitted to an exponential function (red curve, equation, and fit parameters as inset). The purple line represents the final thickness measured $(720 \mathrm{~nm})$. The dashed line represents the calculated depth of an interface at the melting temperature following the equation proposed by Ben-Yakar et al.

agreement with the indicated index-density linear relation. This might indicate that additional processes, not linked to a density change, may occur on the nanosecond time scale, leading to a gradual increase in the refractive index. Little et al. in a similar phosphate glass ${ }^{16}$ found indications for a network modification that locally increases the polarizability of the glass without affecting the local density.

The temporal evolution of the thickness of the HAL is shown in Figure 4. The data are fitted to an exponential function, with the offset being fixed to the value of the final layer thickness $(720 \mathrm{~nm})$. The fit yields a characteristic decay time $\tau=250 \pm 20 \mathrm{~ns}$ and approaches the final value at $4 \tau=1 \mu \mathrm{s}$. Considering the data shown in Figure 4 and Table I, we propose the following scenario of laser excitation and material response to occur: A molten layer (or more precisely, a layer of very low viscosity) is generated after electron-lattice thermalization. Under the high excitation conditions present, the layer reaches temperatures near the boiling temperature. Its transient density (and thus refractive index) is significantly reduced due to the material expansion at high temperatures. ${ }^{17}$ Its initial thickness is, according to the experimental data and extracted from the fit in Figure $4, d_{\text {initial }} \sim 460 \mathrm{~nm}$. The layer has initially a steep temperature profile (also exponential, related to the optical absorption profile), which triggers heat diffusion towards cooler regions underneath and thus melt front propagation into the material.

The above scenario brings up a fundamental question about the nature of the interface of this hot layer with the cooler material. Strictly speaking, no first-order phase transition occurs in glasses upon heating, and therefore, no sharp interface between regions with different structure is expected. Yet, the existence of an interface between material states with different refractive indices is demonstrated by the experimental data. We believe that the interface responsible for optical interference consists in abrupt density gradient caused by an inward propagating rarefaction wave, similar to the one reported in Refs. 13 and 18 but at slower speed. By deriving the fitted exponential function, instantaneous melt front propagation speeds of $1 \mathrm{~m} / \mathrm{s}, 0.3 \mathrm{~m} / \mathrm{s}$, and $0.1 \mathrm{~m} / \mathrm{s}$ are obtained for $5 \mathrm{~ns}, 300 \mathrm{~ns}$, and $600 \mathrm{~ns}$ time delay. These values are much below the typical sound velocities in glasses, which demonstrates that we are not monitoring the propagation of a shock front, but a thermal front.

The propagation of a molten interface in femtosecond laserexcited glasses has also be discussed and modeled by BenYakar et al. ${ }^{6}$ They defined this interface as the isothermal that is at the so-called melting temperature $T_{m}$, being the temperature at which glass can be readily formed, corresponding to a viscosity of $10^{3} \mathrm{~Pa}$ s. Using this criterion and assuming an absorption coefficient of $\mathrm{A}=0.4$, their model yielded a melt-in time of around $300 \mathrm{~ns}$ until a maximum depth of $1 \mu \mathrm{m}$ for their case of borosilicate glass values that are not very different from ours. We have applied their model to calculate melt front propagation for our phosphate glass and our experimental conditions, by solving the equation proposed in Ref. 6, which expresses $T_{m}$ as a function of depth and time assuming a value for $\mathrm{A} \sim 0.5$. Using a value for $n_{e}=1.1 \times 10^{22} \mathrm{~cm}^{-3}$, we obtain $110 \pm 10 \mathrm{~nm}$ as the optical penetration depth. As material parameters, we have used $T_{m}=950 \mathrm{~K}$ (Ref. 19) and those provided by the manufacturer at room temperature $\left(\rho=2700 \mathrm{~kg} \mathrm{~m}^{-3}\right.$, specific heat capacity $c_{p}=810 \mathrm{~J} \mathrm{~kg}^{-1} \mathrm{~K}^{-1}$, and thermal conductivity $k=0.85 \mathrm{~W} \mathrm{~m}^{-1} \mathrm{~K}^{-1}$ ). The result is included in Figure 4 as a dashed line, featuring a poor fit to the experimental data, most likely due to the unknown temperature dependence of the many parameters involved. In view of this complexity, our experimental method to visualize and temporally resolve the formation of the HAL appears as a powerful alternative.

In summary, we have developed a time-resolved imaging technique that is capable of measuring the propagation of a hot viscous layer formed in dielectrics upon femtosecond laser irradiation. Image analysis combined with optical modelling and surface topography measurements allowed us to obtain data of the thickness and refractive index of the heat-affected layer (HAL) as a function of time. The melt-in process follows an exponential decay function, reaching a maximum thickness of the HAL of $720 \mathrm{~nm}$ within around $1 \mu \mathrm{s}$. The final structure of the HAL shows surface swelling without ablation accompanied by a decrease of the refractive index, both consistent with a density decrease throughout the HAL. Already during the melt-in process, a transient decrease in the refractive index and thus decrease in density is observed, consistent with liquid expansion at high temperatures. This finding demonstrates that the refractive index of the final structure is not only a result of the solidification process, but influenced strongly already upon melt-in. Our results corroborate the importance of thermal effects in processing with ultrashort laser pulses, despite the generally accepted idea of non-thermal laser-matter interaction. In terms of applications, the method provides a means to assess the role of the laser repetition rate in laser processing applications where multiple pulses are involved, as it allows determining if following pulses face a still liquid or an already resolidified material.

This work has been partially supported by the Spanish TEC2014-52642-C2-1-R project. M.G.-L. acknowledges the FPU grant awarded by the Spanish Ministry of Education.

${ }^{1}$ R. Osellame, G. Cerullo, and R. Ramponi, Femtosecond Laser Micromachining: Photonic and Microfluidic Devices in Transparent Materials (Springer, 2012).

${ }^{2}$ M. Lebugle, N. Sanner, N. Varkentina, M. Sentis, and O. Utéza, J. Appl. Phys. 116, 063105 (2014). 
${ }^{3}$ M. Garcia-Lechuga, J. Siegel, J. Hernandez-Rueda, and J. Solis, J. Appl. Phys. 116, 113502 (2014).

${ }^{4}$ M. Lenzner, J. Krüger, S. Sartania, Z. Cheng, C. Spielmann, G. Mourou, W. Kautek, and F. Krausz, Phys. Rev. Lett. 80, 4076 (1998).

${ }^{5}$ W. Kautek and J. Krueger, Proc. SPIE 2207, 600 (1994).

${ }^{6}$ A. Ben-Yakar, A. Harkin, J. Ashmore, R. L. Byer, and H. A. Stone, J. Phys. D: Appl. Phys. 40, 1447 (2007).

${ }^{7}$ J. Hernandez-Rueda, J. Siegel, M. Garcia-Lechuga, and J. Solis, J. Opt. Soc. Am. B 31, 1676 (2014).

${ }^{8}$ F. Baset, K. Popov, A. Villafranca, J.-M. Guay, Z. Al-Rekabi, A. E. Pelling, L. Ramunno, and R. Bhardwaj, Opt. Express 21, 12527 (2013).

${ }^{9}$ C. Florian, F. Caballero-Lucas, J. M. Fernández-Pradas, S. Bosch, J. L. Morenza, and P. Serra, J. Phys. D: Appl. Phys. 48, 335302 (2015).

${ }^{10}$ M. C. Downer, R. L. Fork, and C. V. Shank, J. Opt. Soc. Am. B 2, 595 (1985).

${ }^{11}$ J. M. Liu, Opt. Lett. 7, 196 (1982).
${ }^{12}$ D. Puerto, J. Siegel, A. Ferrer, J. Hernandez-Rueda, and J. Solis, J. Opt. Soc. Am. B 29, 2665 (2012).

${ }^{13}$ M. Garcia-Lechuga, J. Siegel, J. Hernandez-Rueda, and J. Solis, Appl. Phys. Lett. 105, 112902 (2014).

${ }^{14}$ M. Born and E. Wolf, Principles of Optics: Electromagnetic Theory of Propagation, Interference and Diffraction of Light (Cambridge University Press, 1997).

${ }^{15}$ J. Siegel, D. Puerto, W. Gawelda, G. Bachelier, J. Solis, L. Ehrentraut, and J. Bonse, Appl. Phys. Lett. 91, 082902 (2007).

${ }^{16}$ D. J. Little, M. Ams, P. Dekker, G. D. Marshall, and M. J. Withford, J. Appl. Phys. 108, 033110 (2010).

${ }^{17}$ W. D. Drotning, Int. J. Thermophys. 6, 705 (1985).

${ }^{18}$ K. Sokolowski-Tinten, J. Bialkowski, A. Cavaleri, D. Von Der Linde, A. Oparin, J. Meyer-ter-Vehn, and S. I. Anisimov, Phys. Rev. Lett. 81, 224 (1998).

${ }^{19}$ A. J. Parsons, N. Sharmin, S. I. S. Shaharuddin, and M. Marshall, J. NonCryst. Solids 408, 76 (2015). 